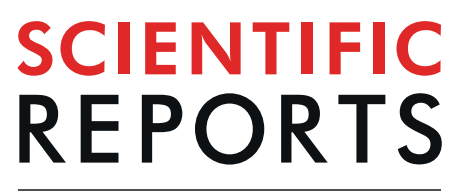

natureresearch

\title{
Multiplex profiling identifies clinically relevant signalling proteins in an isogenic prostate cancer model of radioresistance
}

\author{
S. Inder ${ }^{1,2}$, M. Bates ${ }^{1}$, N. Ni Labhrai ${ }^{1}$, N. McDermott ${ }^{1}$, J. Schneider ${ }^{3}$, G. Erdmann $\mathbb{1}^{3}{ }^{3}$, \\ T. Jamerson ${ }^{4}$, A. N. Flores ${ }^{4}$, A. Prina-Mello ${ }^{5,6}$, P. Thirion ${ }^{7}$, P. R. Manecksha ${ }^{2,8}$, \\ D. Cormican ${ }^{9}$, S. Finn $\mathbb{D}^{9}{ }^{9}$, T. Lynch ${ }^{2}$ \& L. Marignol $\mathbb{1}^{1 *}$
}

The exact biological mechanism governing the radioresistant phenotype of prostate tumours at a high risk of recurrence despite the delivery of advanced radiotherapy protocols remains unclear. This study analysed the protein expression profiles of a previously generated isogenic $22 \mathrm{Rv} 1$ prostate cancer model of radioresistance using DigiWest multiplex protein profiling for a selection of 90 signalling proteins. Comparative analysis of the profiles identified a substantial change in the expression of 43 proteins. Differential PARP-1, AR, p53, Notch-3 and YB-1 protein levels were independently validated using Western Blotting. Pharmacological targeting of these proteins was associated with a mild but significant radiosensitisation effect at 4Gy. This study supports the clinical relevance of isogenic in vitro models of radioresistance and clarifies the molecular radiation response of prostate cancer cells.

Prostate tumours not controlled by radiation therapy ${ }^{1}$ may present with radiation protective biological characteristics $^{2}$ whose pre-treatment identification has the potential to predict treatment outcomes and initiate the development of novel, more aggressive, treatment options. Isogenic models of radioresistance are emerging as clinically-relevant models for the study of these tumours characteristics ${ }^{3}$. This approach has been particularly useful in the characterisation of the radiation-induced DNA damage response $e^{4,5}$. But all identified capabilities or hallmarks of cancer cells can help explain the radiobiological response of tumours ${ }^{3}$. As a result, the signalling pathways known to regulate several cancer hallmarks, such as p53 and Notch ${ }^{6}$, may be key to the regulation of radioresistant cancer cells fate.

While the role of p53 in the increased survival of prostate cancer cells to fractionated radiation ${ }^{7}$, increased cell proliferation ${ }^{8}$ and treatment outcomes ${ }^{9}$ in patients with locally recurrent prostate carcinoma after radiation therapy has been documented, implications of the Notch pathway in the radiation response ${ }^{10}$ is not reported in prostate tumours. The Notch pathway is implicated in angiogenesis ${ }^{11,12}$ and has been proposed to facilitate prostatic tumourigenesis ${ }^{13}$, influence the outcome of anti-cancer hormonal ${ }^{14,15}$ and docetaxel treatments ${ }^{16}$ and may be particularly involved in the development of prostate cancer in men with high body mass index ${ }^{17,18}$. Investigation into the regulation of this pathway indicates a possible cross talk with the YB-1 pathway ${ }^{19,20}$. YB-1 is a multifunctional protein whose expression increases with prostate cancer progression and is predictive of recurrence following surgery ${ }^{21}$. It is involved in both the transcriptional and translational regulation of gene expression, and controls almost all DNA and mRNA dependent processes in the cell such as cellular differentiation, proliferation and stress response ${ }^{22}$.

\footnotetext{
${ }^{1}$ Translational Radiobiology and Molecular oncology, Applied Radiation Therapy Trinity, Trinity Translational Medicine Institute (TTMI), Trinity College Dublin, Dublin, Ireland. ${ }^{2}$ Department of Urology, St James's Hospital, Dublin, Ireland. ${ }^{3}$ NMITT Pharmaservices, Berlin, Germany. ${ }^{4}$ Department of International Health, Mount Sinai School of Medicine, New York, USA. ${ }^{5}$ Laboratory for Biological Characterization of Advanced Materials (LBCAM), Trinity Translational Medicine Institute (TTMI), AMBER centre at CRANN Institute, Trinity College Dublin, Dublin, Ireland. ${ }^{6}$ Department of Clinical Medicine, School of Medicine, Trinity College Dublin, Dublin, Ireland. ${ }^{7}$ St Luke's Radiation Oncology Network, St James's Hospital, Dublin, Ireland. ${ }^{8}$ Department of Surgery, Trinity College Dublin, Dublin, Ireland. ${ }^{9}$ Department of Histopathology, St James's Hospital, Dublin, Ireland. *email: marignl@tcd.ie
} 


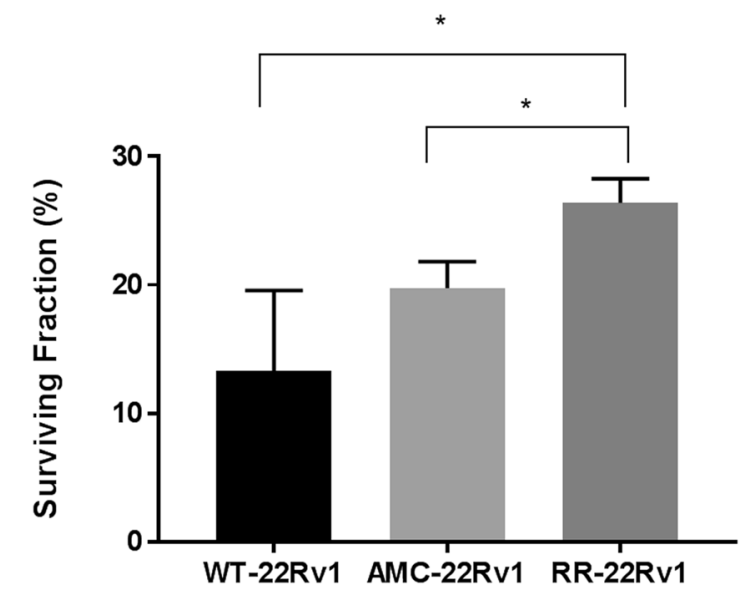

Figure 1. Radiation response of isogenic $22 \mathrm{Rv} 1$ cells. The clonogenic survival of wild type (WT), age-matched controls (AMC) and radioresistant (RR) 22Rv1 prostate cancer cells following a single dose of 4 Gy radiation is presented. $\mathrm{N}=4 ; \mathrm{p}<0.05$.

In prostate cancer, exposure to fractionated radiation progressively selected for a 22Rv1 prostate carcinoma cell population enriched in S-phase cells, less susceptible to DNA damage, radiation-induced apoptosis and acquired enhanced migration potential, when compared to wild type and aged-matched control $22 \mathrm{Rv} 1 \mathrm{cells}^{23}$. These enhanced radioprotective oncogenic properties, also observed in isogenic models of other disease sites ${ }^{3}$, were associated with an altered miRNA profile common to that of 22Rv1 cells exposed to hypoxia, a known factor associated with radioresistance ${ }^{24,25}$. This study aimed to further establish the clinical relevance of the model and identify candidate markers of radioresistance for this disease. Ninety proteins associated with the cancer hallmarks, the Notch and the YB-pathways were selected to generate a custom multiplex protein expression profile of radioresistant (RR-22Rv1) and radiosensitive (WT-22Rv1) isogenic prostate cancer cells. Independent validation of differentially expressed PARP-1, p53 and the androgen receptor strengthens the clinical relevance of the model and suggests a role for the Notch-3 intracellular domain (N3ICD) in the radioresponse of these cells. Pilot analysis in pre-treatment biopsies of prostate cancer patients treated with radiation therapy for the first time implicates the YB-1 protein in treatment failure.

\section{Results}

Radiation response of $22 \mathrm{Rv} \mathbf{1}$ isogenic cells. The change in the radiation response of $22 \mathrm{Rv} 1$ cells exposed to $30 \times 2 \mathrm{~Gy}$ - dose fractions (RR-22Rv1), compared to age-matched (AMC-22Rv1) and wild type (WT-22Rv1) cells was confirmed using clonogenic assays. The clonogenic survival of each cell line treated with a $4 \mathrm{~Gy}$ single dose and their corresponding unirradiated controls is presented in Fig. 1. With a mean survival of $26.4 \% \pm 0.01$, RR-22Rv1 cells were significantly more radioresistant than both AMC-22Rv1 $(18.4 \% \pm 0.01)$ and WT-22Rv1 $(10.31 \% \pm 0.01)$ cells. AMC-22Rv1 showed a non-significant trend towards increased radioresistance, when compared to WT-22Rv1 cells.

Protein profile of the 22Rv1 cells panel. The protein profiles of the isogenic radioresistant cell line (RR-22Rv1) and its parent WT-22Rv1 cell lines were generated for a custom 90 proteins (Fig. 2). Comparison of these profiles identified significant modifications in expression levels for 23 proteins (14 up, 9 down), non-significant trends for 41 (20 up, 21 down) (Table 1), and failed protein detection for 23 (Table 2). The upregulated proteins were associated with the promotion of cell survival, proliferation and invasion (p53, ATR, FKBP12, Bak, Bcl-xL, Beclin-1, Calveolin-1, plasmamix Na+/K+ -ATPase, Claudin-1, Chk1-p-Ser296), the activation of Notch-3 (Notch3 intracellular domain (N3ICD), HES-1) and YB-1 (YB-1, YB-1-p-Ser1-2) signalling. The down regulated proteins were associated with genome instability (plasmamix Histon H3 (diMeth K9), Histone H3), the regulation of tumour growth (Androgen receptor, beta-catenin, Bim, Shh), DNA repair (PARP-1) and Notch signaling (Notch 1 intracellular domain (N1ICD), RBPSUH).

Determination of the expression levels of PARP-1 and the androgen receptor in independent sets of WT-22Rv1, AMC-22Rv1 and RR-22Rv1 validated the decreased expression of these two proteins in cells with a radioresistant phenotype (Fig. 3).

Validation of the p53 expression profile. The expression levels of the p53 protein were confirmed to increase in radioresistant compared to wild type cells in independent cell lysates (Fig. 4A). The expression levels of the p53 pathway regulatory miRNAs, miR-141 and miR-1285 were measured by qPCR in the cell lines. While expression levels of miR-141 were significantly downregulated in RR-22Rv1 and AMC-22Rv1 compared to WT-22Rv1 cells, no significant change in miR-1285 was observed (Fig. 4). The commercially available p53 inhibitor Pifithrin- $\alpha$ (PFT- $\alpha$ ) was next evaluated for its ability to affect the clonogenic survival of the cell lines. WT and RR-22Rv1 cells were treated with two separate concentrations of PFT- $\alpha(20 \mu \mathrm{M}, 40 \mu \mathrm{M}, 2 \mathrm{~h})$ alone or in combination with radiation (4 Gy single dose) (Fig. 4). The surviving fractions of WT-22Rv1 cells treated with either $20 \mu \mathrm{M}(38.7 \pm 0.04)$ or $40 \mu \mathrm{M}(37.5 \% \pm 0.03)$ PFT- $\alpha$ alone were significantly higher than those of cells treated with 
A

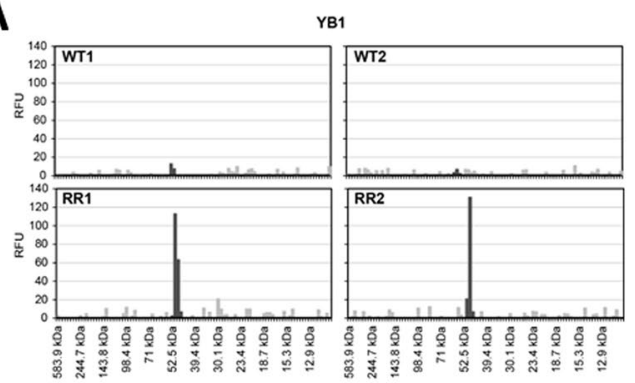

C
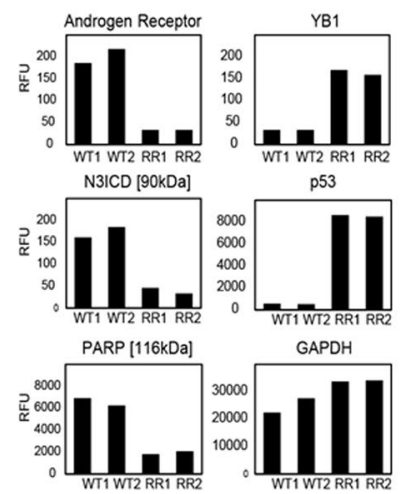

D

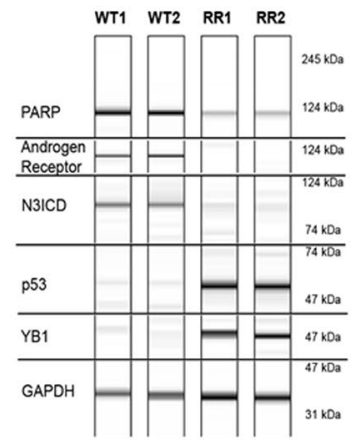

B

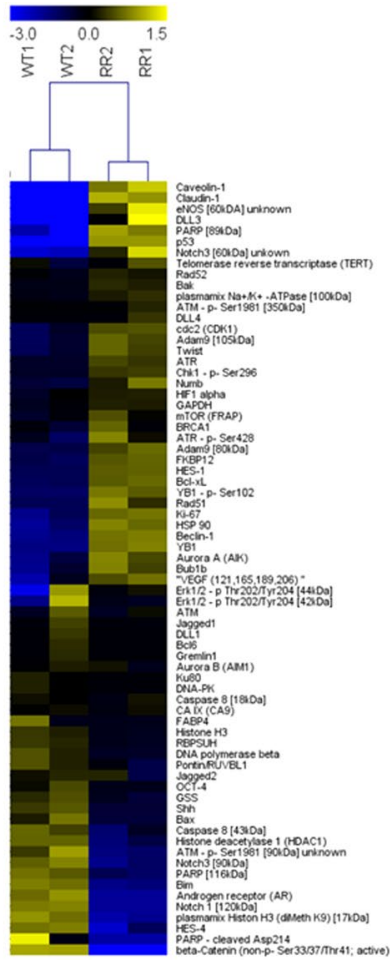

Figure 2. DigiWest protein profiling of wildtype (WT) and radioresistant (RR) 22Rv1 cell lines. (A) Typical representation of DigiWest raw data showing signals for each molecular weight group. Peak signals at the correct molecular weight are marked and quantified in Relative Fluorescence Units (RFU). (B) Heat map of $\log 2$ transformed DigiWest data in WT and RR cell lines. Hierarchical Clustering was performed using complete linkage and Eucledian distance, applying the Multi Experiment Viewer (MeV) software. (C) Bar graphs for DigiWest data from selected proteins in WT vs RR cells. (D) Representation of DigiWest data as Western Blot mimics.

radiation alone $(10.31 \% \pm 0.01)(\mathrm{P}<0.05)$. The combination of PFT- $\alpha$ and $4 \mathrm{~Gy}$ resulted in a significant reduction in surviving fraction when compared to PFT- $\alpha$ alone at both $20 \mu \mathrm{M}(12.2 \% \pm 0.02)$ and $40 \mu \mathrm{M}(5.87 \% \pm 0.01)$ $(\mathrm{p}<0.05)$. The surviving fraction of cells exposed to the combined treatment was significantly reduced at $40 \mu \mathrm{M}$ $(\mathrm{p}<0.05)$, indicating a very small radiosensitising effect of PFT- $\alpha$ at $40 \mu \mathrm{M}$. RR-22Rv1 cells were significantly more sensitive to PFT- $\alpha$ than WT-22Rv1 at both concentrations alone or in combination with radiation $(\mathrm{p}<0.05)$. The surviving fraction of RR-22Rv1 cells $(22 \% \pm 0.1)$ treated with either PFT- $\alpha$ concentration or 4 Gy alone were not significantly different. In response to the combination of PFT- $\alpha$ and $4 \mathrm{~Gy}$, a very small but significant radiosensitising effect was observed as a reduction in clonogenic survival at both concentrations $(p<0.05)$.

Validation of the Notch-3 expression profile. The independent validation of the expression of the Notch-3 receptor indicated strong expression of the cleaved intracellular domain of the Notch-3 receptor (N3ICD) in WT-22Rv1, AMC-22Rv1 and RR-22Rv1 cells. The expression of the full-length receptor appeared to increase in AMC-22Rv1 and RR-22Rv1 cells (Fig. 5A). Evaluation of the cellular localization of N3ICD following radiation exposure ( $5 \mathrm{~Gy}$ ) identified a loss of expression in irradiated WT-22Rv1 prostate cancer cells, but maintained HES-1 expression (Fig. 5B). Treatment of WT-22Rv1 and RR-22Rv1 cells with two commercially available Notch inhibitors 3,5-Difluorophenacetyl)-L-alanyl]-S-phenylglycine t-butyl ester (DAPT) and Batimastat $(60 \mu \mathrm{M})$ failed to significantly decrease clonogenic survival when compared to untreated controls. When the inhibitors were combined with a single $4 \mathrm{~Gy}$ radiation dose, a mild radiosensitising effect was observed in RR-22Rv1 treated with DAPT, with a reduction in clonogenic survival from $28.3 \% \pm 4.4$ (radiation alone) to $11.1 \% \pm 1.1(\mathrm{p}<0.05)$ (Fig. 5C).

Validation of the YB-1 expression profile. The independent validation of the expression of the YB-1 protein indicated strong cytoplasmic and nuclear expression in all three cell lines, with a slight increase in nuclear expression following 4Gy-irradiation (Fig. 6A). Immunostaining for YB-1 was next performed in a series of 22 formalin-fixed parafilm-embedded biopsy specimens from prostate cancer patients. Nuclear expression was detected in 3 out of 4 specimen obtained from patients diagnosed with biochemical failure following radiation therapy. Nuclear expression was not detected in any biopsy specimens from the patients in remission five years post treatment $(n=18)$ (Fig. 7). Treatment of RR-22Rv1 cells with the YB-1 inhibitor Fisetin $(60 \mu M, 24 h)$ resulted in a significant reduction in surviving fraction in unirradiated and 4-Gy irradiated cells (Fig. 6D). The combination of Fisetin with radiation was associated with a reduction in clonogenic survival from $28.4 \% \pm 4.4$ (radiation alone) to $8.5 \% \pm 5.1(\mathrm{p}<0.05)$. 


\begin{tabular}{|c|c|c|c|c|c|c|}
\hline & Analyte & Pathway/Function & Mean WT & Mean RR & $\begin{array}{l}\text { Relationship } \\
\text { between RR and } \\
\text { WT }\end{array}$ & $\begin{array}{l}\text { T.Test } \\
\text { (Log } 2 \\
\text { Values) }\end{array}$ \\
\hline \multicolumn{7}{|c|}{ Proteins with a significant increase in expression in RR-22Rv1 compared to WT-22Rv1 } \\
\hline 1 & plasmamix $\mathrm{Na}+/ \mathrm{K}+-\mathrm{A}$ TPase $[100 \mathrm{kDa}]$ & Intracellular pumps & 178.5 & 258.0 & + & 0.0305 \\
\hline 2 & $\begin{array}{l}\text { Notch3 intracellular domain [(N3ICD) } \\
90 \mathrm{kDa}]\end{array}$ & Notch Signalling/Development & 173.0 & 1642.5 & + & 0.0386 \\
\hline 3 & ATR & Sense DNA damage/Cell cycle arrest & 33.3 & 63.5 & + & 0.0389 \\
\hline 4 & Bak & Apoptosis & 33.3 & 48.0 & + & 0.0364 \\
\hline 5 & Bcl-xL & Apoptosis & 1176.5 & 3718.0 & + & 0.0011 \\
\hline 6 & Beclin-1 & Autophagy & 111.0 & 560.0 & + & 0.0193 \\
\hline 7 & Caveolin-1 & Vesicular trafficking/Cell adhesion/Apoptosis & 33.3 & 3459.0 & + & 0.0217 \\
\hline 8 & Chk1 - p- Ser296 & DNA Damage & 33.3 & 61.0 & + & 0.0173 \\
\hline 9 & Claudin-1 & Cytoskeletal regulation/Adhesion & 73.5 & 4148.5 & + & 0.0031 \\
\hline 10 & FKBP12 & TGF-beta/Smad signaling & 6081.0 & 18052.0 & + & 0.0279 \\
\hline 11 & HES-1 & Transcription Regulation (Supression) & 33.3 & 102.0 & + & 0.0056 \\
\hline 12 & p53 & Cell cycle control/Tumor suppressor & 550.5 & 8581.5 & + & 0.0052 \\
\hline 13 & YB1 & PI3K/Akt Signaling & 33.3 & 164.5 & + & 0.0133 \\
\hline \multirow[t]{2}{*}{14} & YB1 - p- Ser102 & $\begin{array}{l}\text { Transcription factor, RNA metabolism, } \\
\text { Protein Synthesis }\end{array}$ & 33.3 & 122.0 & + & 0.0282 \\
\hline & Analyte & Pathway/Function & Mean WT & Mean RR & $\begin{array}{l}\text { Relationship } \\
\text { between RR and } \\
\text { WT }\end{array}$ & $\begin{array}{l}\text { T.Test } \\
\text { (Log 2 } \\
\text { Values) }\end{array}$ \\
\hline \multicolumn{7}{|c|}{ Proteins with a significant decrease in expression in RR-22Rv1 compared to WT-22Rv1 } \\
\hline 1 & plasmamix Histon H3 (diMeth K9) [17 kDa] & Chromatin regulation/Epigenetics & 40142.0 & 6217.0 & - & 0.0059 \\
\hline 2 & Notch 1 intracellular domain [120kDa] & Notch Signalling & 201.5 & 33.3 & - & 0.0044 \\
\hline 3 & PARP-1 [116kDa] & Apoptosis, DNA damage & 6580.5 & 1944.5 & - & 0.0078 \\
\hline 4 & Androgen receptor (AR) & Nuclear Receptor & 202.0 & 33.3 & - & 0.0281 \\
\hline 5 & $\begin{array}{l}\text { beta-Catenin (non-p- Ser33/37/Thr41; } \\
\text { active) }\end{array}$ & WNT Signalling & 3070.0 & 33.3 & - & 0.0021 \\
\hline 6 & Bim & Apoptosis & 722.5 & 148.0 & - & 0.005 \\
\hline 7 & RBPSUH & Development & 2207.5 & 1355.0 & - & 0.0136 \\
\hline 8 & Shh & Development & 73.5 & 33.3 & - & 0.0494 \\
\hline \multirow[t]{2}{*}{9} & Histone H3 & Chromatin regulation/Epigenetics & 1133292.5 & 721462.5 & - & 0.0452 \\
\hline & Analyte & Pathway/Function & Mean WT & Mean RR & $\begin{array}{l}\text { Relationship } \\
\text { between WT } \\
\text { and RR }\end{array}$ & $\begin{array}{l}\text { T.Test } \\
\text { (Log 2 } \\
\text { Values) } \\
\end{array}$ \\
\hline \multicolumn{7}{|c|}{ Proteins with a non- significant increase in expression in RR-22Rv1 compared to WT-22Rv1 } \\
\hline 1 & ATM - p- Ser1981 [350kDa] & Cell cycle arrest/DNA Repair/Apoptosis & 33.3 & 37.7 & + & 0.5 \\
\hline 2 & ATR - p-Ser428 & Sense DNA damage/Cell cycle arrest & 69.0 & 164.0 & + & 0.138 \\
\hline 3 & Aurora A (AIK) & Cell Cycle Checkpoint & 47.2 & 166.5 & + & 0.1106 \\
\hline 4 & BRCA1 & DNA Damage & 40.7 & 65.5 & + & 0.2946 \\
\hline 5 & Bublb & Cell cycle/Checkpoint & 209.5 & 679.5 & + & 0.1633 \\
\hline 6 & cdc2 (CDK1) & Cell cycle/Checkpoint & 961.0 & 2321.0 & + & 0.1355 \\
\hline 7 & DLL3 & Development & 33.3 & 663.0 & + & 0.1192 \\
\hline 8 & DLL4 & Development & 33.3 & 39.7 & + & 0.5 \\
\hline 9 & GAPDH & Glucose metabolism & 25021.5 & 33990.5 & + & 0.2037 \\
\hline 10 & HIF1 alpha & Protein translation & 41.2 & 56.5 & + & 0.3317 \\
\hline 11 & HSP 90 & Protein folding/Chaperones & 312.0 & 1470.0 & + & 0.0636 \\
\hline 12 & Ki-67 & Proliferation/Tumor marker & 681.0 & 2752.0 & + & 0.0943 \\
\hline 13 & mTOR (FRAP) & mTOR Signalling & 565.0 & 884.5 & + & 0.171 \\
\hline 14 & Numb & Development & 168.5 & 397.5 & + & 0.1339 \\
\hline 15 & Rad51 & DNA Damage & 33.3 & 106.5 & + & 0.1076 \\
\hline 16 & Rad52 & DNA Damage & 300.5 & 398.5 & + & 0.1806 \\
\hline 17 & Telomerase reverse transcriptase (TERT) & Replication & 47.2 & 68.0 & + & 0.4206 \\
\hline 18 & Twist & Transcription & 124.0 & 316.0 & + & 0.0603 \\
\hline 19 & VEGF $(121,165,189,206)$ & Growth Factors/Cytokines & 69.2 & 210.0 & + & 0.267 \\
\hline \multirow[t]{2}{*}{20} & Adam9 [105kDa] & Cytoskeletal Signaling & 101.0 & 263.0 & + & 0.0762 \\
\hline & Analyte & Pathway/Function & Mean WT & Mean RR & $\begin{array}{l}\text { Relationship } \\
\text { between WT } \\
\text { and RR }\end{array}$ & $\begin{array}{l}\text { T.Test } \\
\text { (Log 2 } \\
\text { Values) }\end{array}$ \\
\hline
\end{tabular}




\begin{tabular}{|c|c|c|c|c|c|c|}
\hline & Analyte & Pathway/Function & Mean WT & Mean RR & $\begin{array}{l}\text { Relationship } \\
\text { between RR and } \\
\text { WT }\end{array}$ & \begin{tabular}{|l|} 
T.Test \\
(Log 2 \\
Values)
\end{tabular} \\
\hline \multicolumn{7}{|c|}{ Proteins with a non-significant decrease in expression in RR-22Rv1 compared to WT-22Rv1 } \\
\hline 1 & Erk1/2 - p Thr202/Tyr204 [42kDa] & MAPK Signalling & 817.0 & 512.0 & - & 0.716 \\
\hline 2 & Caspase $8[43 \mathrm{kDa}]$ & Apoptosis & 123.0 & 47.2 & - & 0.1704 \\
\hline 3 & OCT 4 & Stem Cell Marker & 52.0 & 33.3 & - & 0.1919 \\
\hline 4 & ATM & Cell cycle arrest/DNA Repair/Apoptosis & 50.7 & 41.2 & - & 0.7351 \\
\hline 5 & Aurora B (AIM1) & Cell Cycle Checkpoint & 382.0 & 334.5 & - & 0.5505 \\
\hline 6 & Bax & Apoptosis & 77.0 & 33.3 & - & 0.1403 \\
\hline 7 & Bcl6 & Lymphocytes Signalling & 38.7 & 33.3 & - & 0.5 \\
\hline 8 & CA IX (CA9) & Hypoxia Biomarker & 80.0 & 73.5 & - & 0.5744 \\
\hline 9 & DLL1 & Development & 40.2 & 33.3 & - & 0.5 \\
\hline 10 & DNA polymerase beta & DNA Damage & 1857.5 & 1008.0 & - & 0.1013 \\
\hline 11 & DNA-PK & DNA Damage & 98.0 & 83.0 & - & 0.2625 \\
\hline 12 & FABP4 & Metabolism & 51.7 & 33.3 & - & 0.5 \\
\hline 13 & Gremlin1 & Oxidative Stress/Antioxidant & 38.2 & 33.3 & - & 0.5 \\
\hline 14 & GSS & Oxidative Stress & 255.0 & 134.0 & - & 0.0511 \\
\hline 15 & HES-4 & Transcription Regulation & 295.5 & 56.7 & - & 0.156 \\
\hline 16 & Histone deacetylase 1 (HDAC1) & Chromatin regulation/Epigenetics & 140.0 & 42.2 & - & 0.1096 \\
\hline 17 & Jagged1 & Development & 41.7 & 33.3 & - & 0.5 \\
\hline 18 & Jagged2 & Development & 69.5 & 52.7 & - & 0.5304 \\
\hline 19 & Ku80 & DNA Damage & 7350.5 & 6191.0 & - & 0.2563 \\
\hline 20 & PARP - cleaved Asp214 & Apoptosis & 231.0 & 33.3 & - & 0.1993 \\
\hline 21 & Pontin/RUVBL1 & $\begin{array}{l}\text { Chromatin remodeling, regulating gene } \\
\text { transcription }\end{array}$ & 216.5 & 118.5 & - & 0.1912 \\
\hline
\end{tabular}

Table 1. Differential protein expression patterns in RR-22Rv1 compared to WT-22Rv1 cell samples. The relationship to radioresistance is marked with a $(+)$ if expression was increased in $\mathrm{RR}-$ compared to WT22Rv1 cells. A decrease in expression is marked with a (-).

\section{Discussion}

The effects of radiation exposure on cancer cells and subsequent regulation of cell fate has been linked to DNA damage induction ${ }^{4,5}$, all known cancer hallmarks ${ }^{3}$, and the tumour microenvironment ${ }^{26}$. This study proposed to compare the protein expression profiles for a selection of signaling proteins involved in these biological processes in a pre-existing isogenic model of $22 \mathrm{Rv} 1$ prostate cancer cells ${ }^{23}$. High content multiplex protein profiling identified twenty-three proteins within the selected panel whose expression was significantly modified in the radioresistant line, compared to the wild type cell line.

The DNA repair protein PARP-1 was independently identified as one of the protein whose expression was down-regulated in radioresistant cells, alongside the androgen receptor (AR). PARP-1 has been implicated in epithelial-mesenchymal-transition (EMT) during prostate tumorigenesis ${ }^{27}$, and its elevated expression was associated with poor prognosis following radiotherapy ${ }^{28}$. PARP-1 inhibition appears most effective in tumours with elevated PARP-1, but reduced AR and p53 expression levels ${ }^{29,30}$. Our data support these findings and suggest that tumour recurrence following exposure to fractionated radiation may result from the selection of cells with reduced PARP-1 and AR as well as elevated p53 expression. Further evaluation of this molecular combination in radiotherapy patients is warranted.

The expression of p53 was increased in RR-22Rv1 cells and correlated with the downregulation of miR-141. Reports of the upregulation of miR-141 in prostate cancer suggest its involvement in the metastatic process ${ }^{31}$, the regulation of AR activity ${ }^{32}$ and modifications in cell proliferation rates and apoptosis susceptibility ${ }^{33}$, but no previous reports have shown evidence of its involvement in the response to radiation therapy. Treatment of RR-22Rv1 cells with PFT- $\alpha$, a known suppressor of p53 as well as glucocorticoid and heat shock receptor factor ${ }^{34}$ was associated with a very small but significant radiosensitisation effect. This is the first report of a radiosensitisation effect of this compound in prostate cancer and further evaluation of the magnitude of radiosensitisation that may be achieved is warranted.

Our data identifies the up regulation of the intracellular domain of the Notch-3 receptor in RR-22Rv1, which correlates with a mild radiosensitisation effect following exposure to the Notch inhibitor DAPT. Notch inhibitors are increasingly explored as novel anti-cancer strategies ${ }^{35-37}$ and their potential as radiosensitisers has been proposed ${ }^{10}$. We $\mathrm{Wr}^{38,39}$, like others ${ }^{40}$, have previously reported the upregulation of the Notch-3 receptor in prostate cancer, and its role in hypoxic tumours ${ }^{41}$. Although Notch activity has been implicated in the radiation-induced stress response of prostate cancer cells ${ }^{42}$, no such strategy exists in prostate cancer. Exposure to a single $5 \mathrm{~Gy}$ radiation dose led to undetectable expression of the intracellular domain of the Notch-3 receptor in wild type 22Rv1 cells. This loss did not correlate with a reduction in HES-1 expression, a known downstream target of the Notch pathway. This data indicates that the regulation of HES-1 is not dependent on Notch-3 activation. In prostate tumours, HES-1 elevated expression was also related to Notch-1 receptor activation ${ }^{43}$. In prostate cancer cells, while the selective genetic manipulation of members of the pathways (Notch- $1^{44}$ ) impaired differentiation and 


\begin{tabular}{|l|l|l|}
\hline & Protein & Pathway/Function \\
\hline 1 & eNOS [140kDa] & PI3K/Akt Signaling \\
\hline 2 & Notch $1[300 \mathrm{kDa}]$ & Notch Signalling \\
\hline 3 & Bad & Apoptosis \\
\hline 4 & Bcl2 & Apoptosis \\
\hline 5 & CD133 & MAPK signaling/Akt signaling \\
\hline 6 & CD44 & Stem Cell Marker \\
\hline 7 & Chk2 & Cell Cycle/Checkpoint \\
\hline 8 & Cox2 & NF-kB signaling \\
\hline 9 & Dicer1 & Protein Translation \\
\hline 10 & EGFR (ErB-1, HER1) & Tyrosine kinase/Adaptor \\
\hline 11 & Eotaxin & Inflammatory response \\
\hline 12 & FoxO3a - p- Ser413 & PI3K/Akt Signaling \\
\hline 13 & $\begin{array}{l}\text { Notch 1 - cleaved } \\
\text { Val1744 }\end{array}$ & Notch Signalling \\
\hline 14 & Notch 2 & Notch Signalling \\
\hline 15 & $\begin{array}{l}\text { P-Glycoprotein }(\text { MDR1, } \\
\text { ABCB1) }\end{array}$ & Metabolism \\
\hline 16 & PTEN - p- Ser380 & Metabolism/Cell Cycle \\
\hline 17 & Puma & Modulator of Apoptosis \\
\hline 18 & Rad50 & DNA Damage \\
\hline 19 & SFRP2 & Wnt signaling \\
\hline 20 & Sox2 & Stem Cell Marker \\
\hline 21 & Stathmin 1 & Cytoskeletal signaling \\
\hline 22 & Survivin - p- Thr34 & Apoptosis \\
\hline 23 & VE-Cadherin & Adhesion \\
\hline 24 & VEGF-A & Angiogenesis \\
\hline
\end{tabular}

Table 2. List of analysed Proteins that were below detection levels.

A

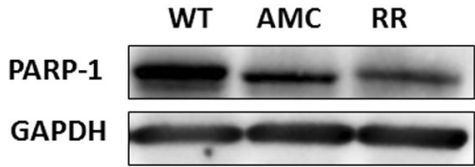

B
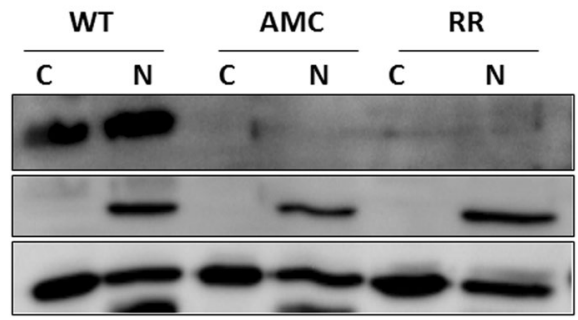

AR

LAMIN B1

GAPDH

Figure 3. Representative cropped immunoblots of wild type (WT), age-matched controls (AMC) and radioresistant (RR) 22Rv1 prostate cancer cells. The detection levels of (A) PARP-1 in whole cell lysates, and (B) the androgen receptor $(A R)$ in the nuclear $(\mathrm{N})$ and cytoplasmic $(\mathrm{C})$ protein fractions are presented. GAPDH or Lamin B1 were used as cytoplasmic and nuclear loading controls respectively. The full length blots are presented in Supplementary Figs. 1A,B and 2A,B.

tumour growth ${ }^{45,46}$, the pharmacological inhibition of the pathway with gamma-secretase inhibitors only caused mild modifications $s^{47,48}$. Examples of Notch targeted radiosensitising strategies are beginning to emerge in lung cancer $^{49}$ and glioblastoma ${ }^{42,50}$.

Finally, the comparative analysis of these protein profiles identifies upregulation of the YB-1 protein. Nuclear localization of YB-1 has been proposed as an indicator of poor prognosis ${ }^{51}$, with high protein levels of YB-1 and MTA1 associated with a 3-fold increased risk for requiring future hormone therapy or radiation therapy in prostate cancer ${ }^{21}$. Our data confirmed detection of the protein in the cytoplasm and nucleus of all three cell lines, and a slight elevation in the RR-22Rv1 cells. Exposure of RR-22Rv1 cells to a 4 Gy single radiation dose resulted in a 
A

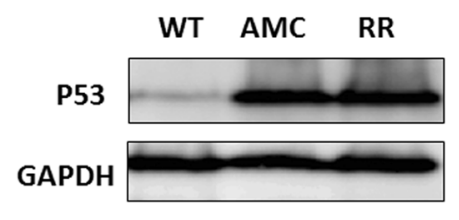

C

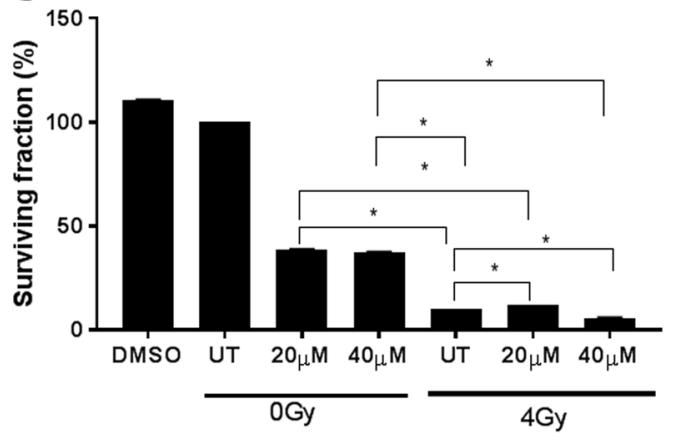

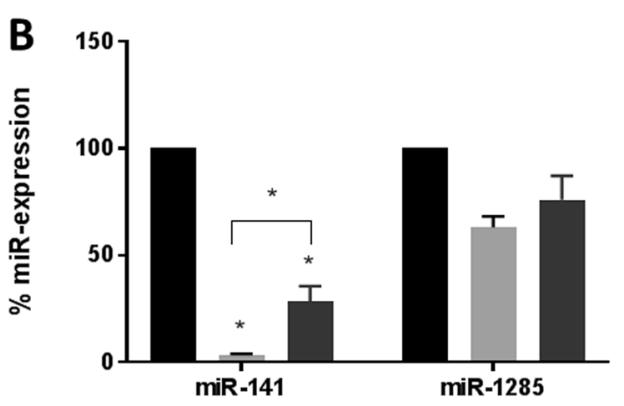

- WT-22Rv1 AMC-22Rv1 - RR-22Rv1

D

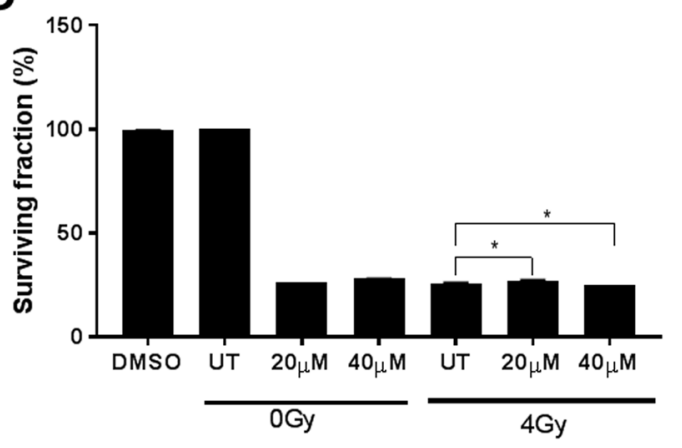

Figure 4. Evaluation of $\mathrm{p} 53$ as a marker of radioresistance. (A) Representative cropped immunoblots for the detection of p53 in wild type (WT), age-matched controls (AMC) and radioresistant (RR) 22Rv1 prostate cancer cells. GAPDH was used as the loading control. Full length blots are presented in Supplementary Fig. 3A,B. (B) miR-141 and miR-1285 expression profiles in WT-, AMC- and RR-22Rv1 prostate cancer cell lines was determined by qPCR. MiR-141 and miR-1285 expression levels for each cell line were normalised to the endogenous control miR-16 and calibrated to that of WT-22Rv1 cells to establish the relative change in microRNA expression (\% miR-expression). Results are expressed as mean $+/-\mathrm{SD}, \mathrm{N}=3$. Clonogenic survival of (C) WT-22Rv1 and (D) RR-22Rv1 treated with Pifithrin- $\alpha(20 \mu \mathrm{M}, 40 \mu \mathrm{M}, 2 \mathrm{hrs})$ alone or in combination with a single dose of $4 \mathrm{~Gy}$ radiation. The clonogenic survival of untreated cells was used as a control. UT: untreated controls, $* \mathrm{p}<0.05$.

modest increase in nuclear YB-1 expression levels. Pilot analysis identified elevated nuclear expression in 3 out of 4 formalin-fixed-parafilm-embedded biopsy specimen from high risk radiotherapy prostate cancer patients whose tumours progressed post-treatment and none in those with no evidence of residual disease at five years. The inhibition of YB-1 by Fisetin was associated with microtubule stabilising properties, an ability to reverse mesenchymal features and inhibit cell proliferation, migration, and invasion ${ }^{52}$. Treatment of RR-22Rv1 with this dietary flanovol resulted in a significant reduction in the clonogenic survival of irradiated cells. This is the first report of the radiosensitisation potential of this YB-1 inhibitor.

In conclusion, the exposure of $22 \mathrm{Rv} 1$ prostate cancer cells to fractionated radiation and emergence of a radioresistant cell line is associated with the deregulation of signalling proteins associated with more aggressive disease and poor prognosis following radiotherapy. This work supports the clinical relevance of this model and identifies actionable markers of the radioresistant phenotype of prostate cancer.

\section{Materials and Methods}

Cell culture. Newly acquired, authenticated human 22Rv1 (WT-22Rv1) prostate cancer cells (American Type Culture Collection) were cultured at $37^{\circ} \mathrm{C}$ in $95 \%$ humidified air containing $5 \% \mathrm{CO}_{2}$ in RPMI cell culture medium containing l-glutamine (Lonza, Dublin, Ireland) with 10\% foetal bovine serum (Gibco, Dublin, Ireland) and 1\% pen/strep (Lonza). Fractionated $2 \mathrm{~Gy}$ X-rays doses $(250 \mathrm{keV}, 15 \mathrm{~mA}$ ) using an RS225 cabinet (XStrahl, Surrey, UK) were delivered weekly to a cumulative dose of $60 \mathrm{~Gy}$ to generate the RR-22RV1 cell model, as described in ${ }^{23}$. Mock irradiated cells were cultured alongside to generate age-matched controls cells (AMC-22Rv1) ${ }^{23}$.

Clonogenic assays. Cell survival was evaluated using a standard colony forming assay ${ }^{53}$. Cells were treated with Pifithrin- $\alpha$ (Sigma Aldrich, Dublin, Ireland) at concentrations of $20 \mu \mathrm{M}$ and $40 \mu \mathrm{M}$ for 2 hours; batimastat, DAPT or Fisetin (Sigma Aldrich) at a concentration of $60 \mu \mathrm{M}$ for 24 hours. The cells were then irradiated at a single dose of $4 \mathrm{~Gy}$ and incubated for 7-14 days until formation of colonies. The colonies washed with PBS, were fixed and stained using a $0.05 \%$ Crystal Violet (Sigma Aldrich) in 70\% Methanol solution. Individual colonies (defined as a cluster of greater than 50 cells) were counted using the ColCount software (Oxford Optronix Ltd, Abingdon,UK). 
A

\section{WT AMC RR}
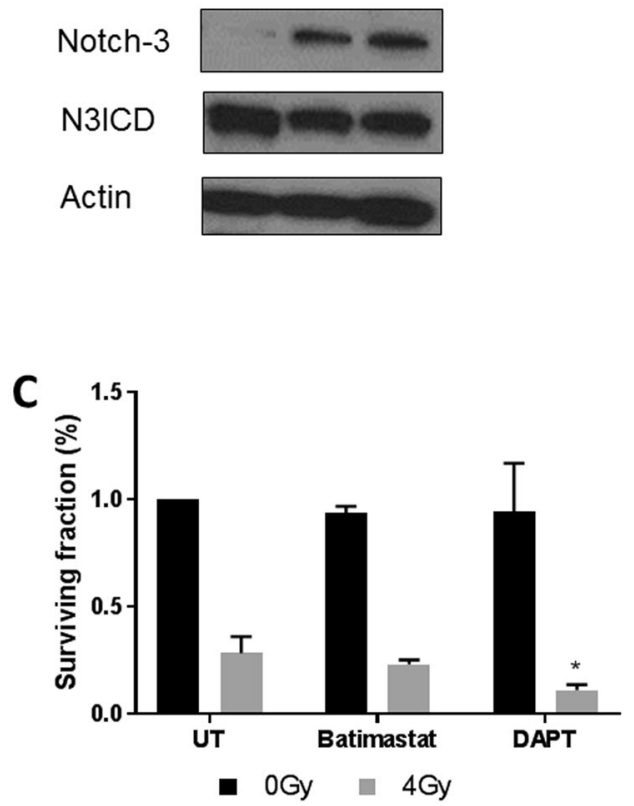

B 22Rv1

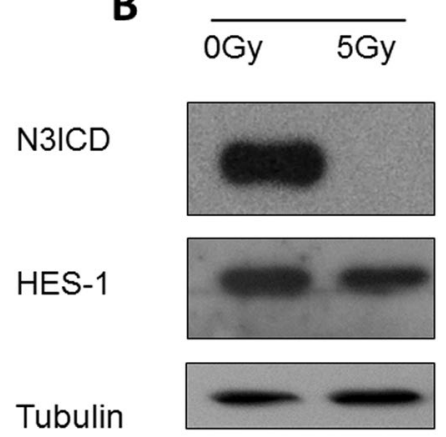

Figure 5. Evaluation of Notch-3 as a marker of radioresistance. (A) Representative cropped immunoblots of Notch-3 and its intracellular domain in wild type (WT), age-matched controls (AMC) and radioresistant (RR) 22Rv1 prostate cancer cells. The full length blots are presented in Supplementary Fig. 4. (B) The detection levels of the Notch-3 intracellular domain (N3ICD) and HES-1 in the protein fractions of untreated and 5Gyirradiated WT-22Rv1 cells are presented. Actin or Tubulin were used as loading controls. The full blots are presented in Supplementary Fig. 5A-C. (C) Clonogenic survival of RR-22Rv1-cells treated with the Notchinhibitors Batimastat or DAPT $(60 \mu \mathrm{M}, 24 \mathrm{hrs})$, alone or in a combination with a $4 \mathrm{~Gy}$ single radiation dose. $* \mathrm{p}<0.05$.

A

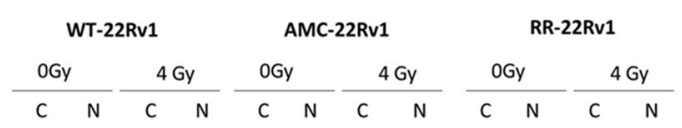

YB-1

Vinculin

Lamin B1
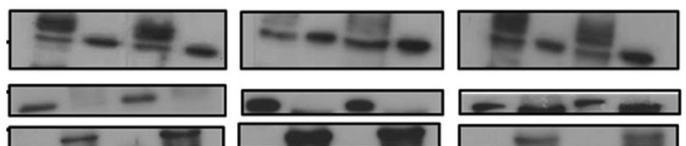

B

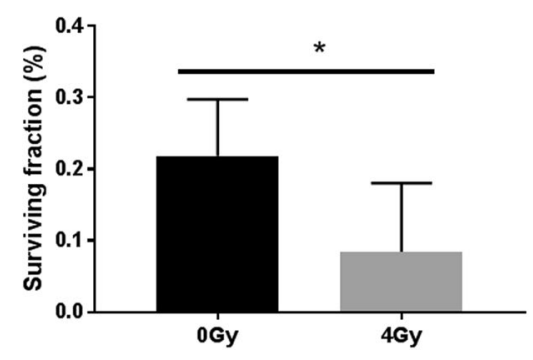

Figure 6. Evaluation of YB-1 as a marker of radioresistance. (A) Representative cropped immunoblots of YB-1 in the cytoplasmic (C) and nuclear (N) protein fractions of wild type (WT), age-matched controls (AMC) and radioresistant (RR) $22 \mathrm{Rv} 1$ prostate cancer cells which received a single dose of $4 \mathrm{~Gy}$ radiation or were left untreated. Vinculin and Lamin B1 were used as cytoplasmic and nuclear loading controls respectively. The full length blots are presented in Supplementary Fig. 6A-C. (B) Clonogenic survival of unirradiated and 4-Gy irradiated RR-22Rv1 cells treated with the YB-1 inhibitor Fisetin $(60 \mu \mathrm{M}, 24 \mathrm{hrs}) .{ }^{*} \mathrm{p}<0.05$. 


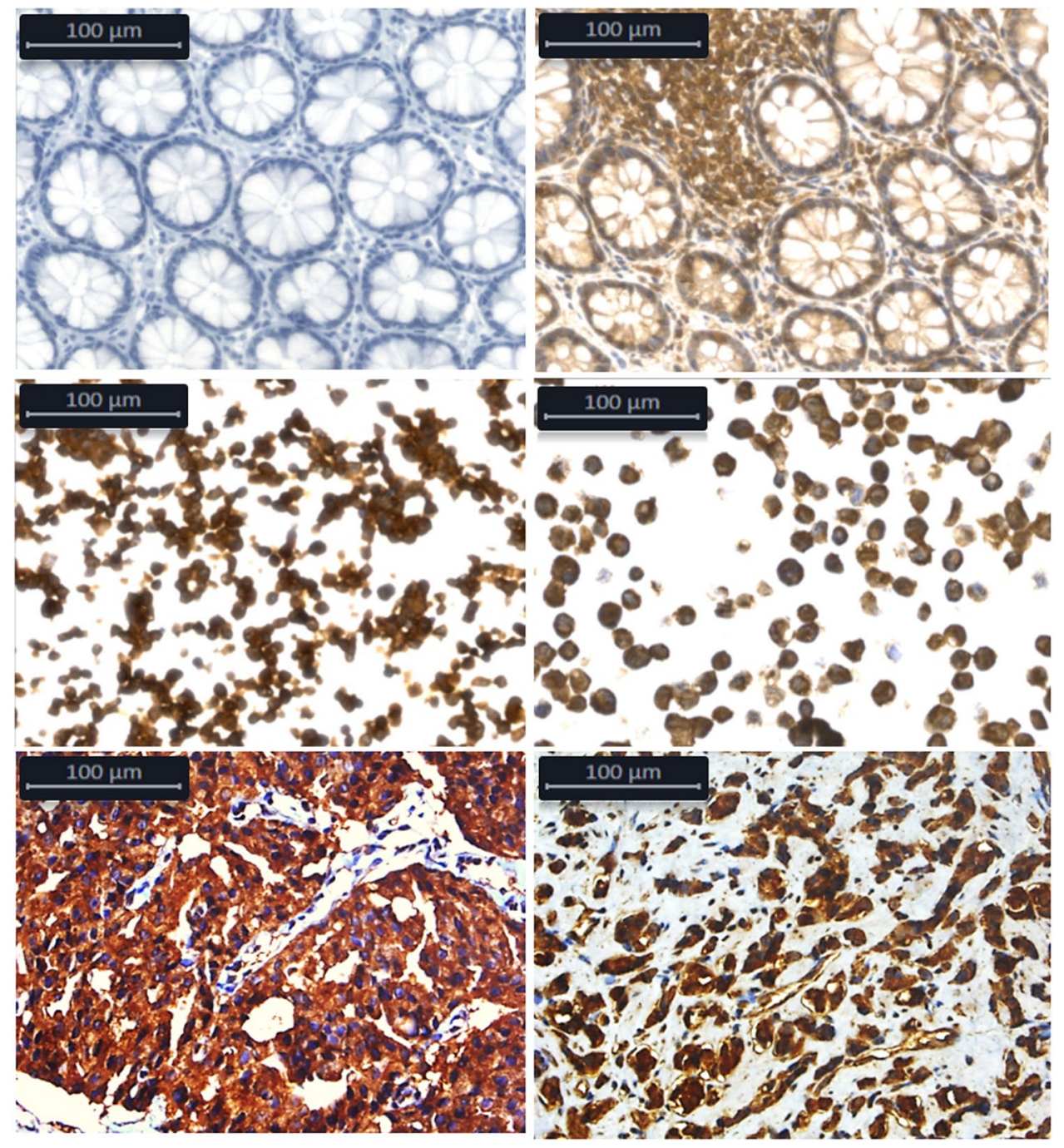

Figure 7. Representative images of positive and negative YB1 staining. Top left - Colon control tissue stained without primary antibody. Top Right - Colon control tissue displaying positive staining. Centre Left - WT22RV1 cells exhibiting predominantly cytoplasmic staining for YB1. Centre right - RR-22RV1 cells which display cytoplasmic and enhanced nuclear YB1 staining. Bottom left - A pre-treatment prostate biopsy from a patient who subsequently underwent radiation therapy and exhibited a favourable response which display predominantly cytoplasmic YB1 staining. Bottom right - A biopsy from a non-responder/radioresistant patient displaying enhanced nuclear YB1 staining. Images were taken at 40X magnification using a Leica DM 3000 Microscope and Leica DFC 425 camera.

DigiWest multiplex protein profiling. DigiWest analyses were performed as previously described ${ }^{54}$. Briefly, gel electrophoresis and Western blotting onto PVDF membranes was conducted using the NuPAGE system (Life Technologies, Carlsbad, USA). Blots were washed in PBST and proteins were biotinylated on the membrane using $50 \mu \mathrm{M}$ NHS-PEG12-Biotin in PBST for $1 \mathrm{~h}$, then washed in PBST and dried. Each sample lane was cut into 96 molecular weight fractions of $0.5 \mathrm{~mm}$ each and proteins were eluted in 96 well plates using per well $10 \mu$ l elution buffer ( $8 \mathrm{M}$ urea, $1 \%$ Triton-X100 in $100 \mathrm{mM}$ Tris-HCl pH 9.5). Eluted proteins from each molecular weight fraction were loaded onto one distinct color of neutravidin coated MagPlex beads (Luminex, Austin, TX, USA) and then pooled. A total of $10 \mu \mathrm{g}$ of protein per cell sample was used for generation of 90 different antibody incubations.

Aliquots of the DigiWest bead mixes were added to 96 well plates containing $50 \mu$ lassay buffer (Blocking Reagent for ELISA (Roche, Rotkreuz, Switzerland) supplemented with 0.2\% milk powder, 0.05\% Tween-20 and $0.02 \%$ sodium azide). After discarding the assay buffer, $30 \mu \mathrm{l}$ of diluted primary antibody was added per well. Following overnight incubation at $15^{\circ} \mathrm{C}$ on a shaker, bead mixes were washed twice with PBST, and Phycoerythrin-labelled secondary antibodies (diluted in assay buffer) were added and incubated for $1 \mathrm{~h}$ at $23^{\circ} \mathrm{C}$. Beads were washed twice prior to readout on a Luminex FlexMAP 3D.

For quantification of antibody specific signals, the DigiWest analysis tool described in $^{54}$ was used to identify peaks and to calculate peak areas. For comparative analyses, protein expression values were normalized to the total protein per lane. 
miRNA expression analysis. Total RNA was isolated as per the manufacturer's instructions using the mirVana ${ }^{\mathrm{TM}}$ miRNA Isolation Kit (Applied Biosystems, USA). RNA concentration was determined using the nanodrop 1000 spectrophotometer. Reverse transcription was carried out using the High Capacity cDNA Reverse Transcription Kit (Applied Biosystems) on the Gene Amp PCR System 9600 (Perkin Elmer). TaqMan RT-PCR was then performed in 384 well plates using the 7900HT Real-Time PCR System (Applied Biosystems) using primers and probes for miR-141, miR-1285 and miR-16 and TaqMan ${ }^{\mathrm{TM}}$ Universal PCR Master Mix, no AmpErase $^{\mathrm{TM}}$ UNG (Thermo Fisher Scientific) mixed together with amplified cDNA samples as per the manufacturer's protocols. MiRNA expression levels were calculated using the $\Delta \Delta \mathrm{CT}$ method ${ }^{55}$ relative to the endogenous control, miR-16. A change in miRNA expression was considered significant if at least a 2 -fold change (above $200 \%$ expression or below $50 \%$ expression) was observed, with a p value of $\leq 0.05$ compared to WT-22Rv1 cells.

Western blotting. Proteins were extracted from cells using either RIPA Lysis buffer (Santa Cruz, USA) or the NE-PER Nuclear and Cytoplasmic Extraction kit (Thermo Fisher Scientific, USA) modified with phenylmethanesulfonyl fluoride (PMSF) $(200 \mathrm{mM})$, a protease inhibitor cocktail, and sodium orthovanadate $(100 \mathrm{mM})$, according to the manufacturer's instructions Protein concentration was determined using the Pierce ${ }^{\mathrm{TM}} \mathrm{BCA}$ Protein Assay Kit ((Thermo Fisher Scientific). $20 \mu \mathrm{g}$ of each protein sample was then resolved by SDS-PAGE on a 5\% stacking gel and $10 \%$ resolving gel using a Mini-Protean Tetra Cell electrophoresis rig (BioRad, USA). Resolved proteins were then transferred to $0.2 \mu \mathrm{M}$ Hybond PVDF membranes (Amersham, UK) using the XCell $\mathrm{II}^{\mathrm{TM}}$ Blot module (Invitrogen, Dublin, Ireland). Following transfer membranes were blocked using $5 \% \mathrm{w} / \mathrm{v}$ milk protein and probed using primary antibodies directed against PARP1 (\#sc-8007; 1:100, Santa Cruz, UK ${ }^{56}$ ), AR (\#sc-377546, 1:250, Santa Cruz), p53 (\#sc-126, 1:500, Santa Cruz, UK ${ }^{57}$ ), Notch-3 (\#ab23426, 1:250, Abcam, UK $^{58}$ ), HES-1 (\#ab71559, 1:1000, Abcam ${ }^{59}$ ) or YB-1 (\#ab12148, 1:2000, Abcam ${ }^{60}$ ). After washing, the membrane was incubated with horseradish peroxidase conjugated goat secondary antibody (sc-2020, 1:1000, Santa $\mathrm{Cruz}^{61}$ ) or Anti-Rabbit IgG HRP Linked (\#7074, 1:1000, Cell Signalling ${ }^{62}$ ) antibodies. Following incubation with the primary and secondary antibodies, a detection reagent luminol (SC-2048, Santa Cruz) was applied to blots and chemiluminescence images were then developed using a Fujifilm LAS-4000 luminescent image analyser. Molecular weight was confirmed using the MagicMark ${ }^{\mathrm{TM}}$ XP Western Protein Standard (LC5602, Thermo Fisher Scientific, Dublin, Ireland). Membranes were stripped prior to reprobing with either anti-actin (1:10000, Sigma-Aldrich ${ }^{63}$ ), anti-GAPDH (\#ab9485, 1:10,000, Abcam ${ }^{63}$ ), Lamin (\#GTX103292, 1:1,000, Genetex, UK ${ }^{64}$ ) or Vinculin (\#GTX113294, 1:1,000, Genetex ${ }^{64}$ ).

Patient specimens. Diagnostic biopsies $(n=22)$ were obtained retrospectively with informed consent from high-risk prostate cancer patients with a primary Gleason score of 8-10 who subsequently underwent androgen deprivation therapy and external beam radiation therapy. Ethical approval was obtained from St James's/ Tallaght Hospital and the St Luke's Radiation Oncology Network, Dublin, Ireland. All research was performed in accordance with relevant guidelines/regulations. Four patients were diagnosed with biochemical failure and disease recurrence, according to the Phoenix criteria. The remaining patients $(n=18)$ were in remission at 5 years post- treatment.

Immunohistochemistry. $\quad 4 \mu \mathrm{M}$ sections were cut from the 22 selected FFPE patient tissue blocks and were baked onto charged slides overnight in an oven at $65^{\circ} \mathrm{C}$ prior to storage until needed. Slides were stained using the Rabbit specific HRP/DAB (ABC) Detection IHC Kit (Abcam, ab64261) and a rabbit polyclonal YB-1 antibody (Abcam, ab12148) diluted 1:100 in PBS. Immunostained sections were blindly examined by two trained histopathologists (SF \& DC). Images were taken using a Nikon Eclipse E200 microscope and OC view 7 software.

Statistical analysis. A Student's t-test or one way ANOVA was used to compare means between treatment and control groups as appropriate. Statistical analysis was performed using Prism, Version 5.01 (GraphPad Software Inc. CA). A p-value of $<0.05$ was considered statistically significant.

\section{Data availability}

The datasets generated during and/or analysed during the current study are available from the corresponding author on reasonable request.

Received: 25 May 2018; Accepted: 4 November 2019;

Published online: 22 November 2019

\section{References}

1. Shao, Y. H. et al. Contemporary risk profile of prostate cancer in the United States. Journal of the National Cancer Institute 101, 1280-1283, https://doi.org/10.1093/jnci/djp262 (2009)

2. Good, J. S. \& Harrington, K. J. The hallmarks of cancer and the radiation oncologist: updating the 5Rs of radiobiology. Clinical oncology 25, 569-577, https://doi.org/10.1016/j.clon.2013.06.009 (2013).

3. McDermott, N., Meunier, A., Lynch, T. H., Hollywood, D. \& Marignol, L. Isogenic radiation resistant cell lines: development and validation strategies. International journal of radiation biology 90, 115-126, https://doi.org/10.3109/09553002.2014.873557 (2014).

4. Gavande, N. S. et al. DNA repair targeted therapy: The past or future of cancer treatment? Pharmacol Ther 160, 65-83, https://doi. org/10.1016/j.pharmthera.2016.02.003 (2016).

5. Goldstein, M. \& Kastan, M. B. The DNA damage response: implications for tumor responses to radiation and chemotherapy. Annu Rev Med 66, 129-143, https://doi.org/10.1146/annurev-med-081313-121208 (2015).

6. Sanchez-Vega, F. et al. Oncogenic Signaling Pathways in The Cancer Genome Atlas. Cell 173, 321-337 e310, https://doi. org/10.1016/j.cell.2018.03.035 (2018).

7. Scott, S. L., Earle, J. D. \& Gumerlock, P. H. Functional p53 increases prostate cancer cell survival after exposure to fractionated doses of ionizing radiation. Cancer research 63, 7190-7196 (2003). 
8. Cheng, L. et al. p53 protein overexpression is associated with increased cell proliferation in patients with locally recurrent prostate carcinoma after radiation therapy. Cancer 85, 1293-1299 (1999).

9. D’Amico, A. V. et al. $\mathrm{p} 53$ protein expression status and recurrence in men treated with radiation and androgen suppression therapy for higher-risk prostate cancer: a prospective phase II Cancer and Leukemia Group B Study (CALGB 9682). Urology 71, 933-937, https://doi.org/10.1016/j.urology.2007.11.005 (2008).

10. Yahyanejad, S., Theys, J. \& Vooijs, M. Targeting Notch to overcome radiation resistance. Oncotarget 7, 7610-7628, https://doi. org/10.18632/oncotarget.6714 (2016).

11. Moya, I. M. et al. Stalk cell phenotype depends on integration of Notch and Smad1/5 signaling cascades. Dev Cell 22, 501-514, https://doi.org/10.1016/j.devcel.2012.01.007 (2012).

12. Sewduth, R. \& Santoro, M. M. "Decoding” Angiogenesis: New Facets Controlling Endothelial Cell Behavior. Front Physiol 7, 306, https://doi.org/10.3389/fphys.2016.00306 (2016).

13. Pedrosa, A. R. et al. Notch signaling dynamics in the adult healthy prostate and in prostatic tumor development. Prostate 76, 80-96, https://doi.org/10.1002/pros.23102 (2016).

14. Villaronga, M. A., Bevan, C. L. \& Belandia, B. Notch signaling: a potential therapeutic target in prostate cancer. Curr Cancer Drug Targets 8, 566-580 (2008).

15. Kong, D. et al. Epigenetic silencing of miR-34a in human prostate cancer cells and tumor tissue specimens can be reversed by BRDIM treatment. American journal of translational research 4, 14-23 (2012).

16. Domingo-Domenech, J. et al. Suppression of acquired docetaxel resistance in prostate cancer through depletion of notch- and hedgehog-dependent tumor-initiating cells. Cancer cell 22, 373-388, https://doi.org/10.1016/j.ccr.2012.07.016 (2012).

17. Sharad, S. et al. Prostate cancer gene expression signature of patients with high body mass index. Prostate Cancer Prostatic Dis 14, 22-29, https://doi.org/10.1038/pcan.2010.44 (2011).

18. Webber, J. et al. Proteomics analysis of cancer exosomes using a novel modified aptamer-based array (SOMAscan) platform. Molecular \& cellular proteomics: MCP 13, 1050-1064, https://doi.org/10.1074/mcp.M113.032136 (2014).

19. Rauen, T. et al. YB-1 acts as a ligand for Notch-3 receptors and modulates receptor activation. J Biol Chem 284, 26928-26940, https:// doi.org/10.1074/jbc.M109.046599 (2009).

20. Toulany, M. et al. Impact of oncogenic K-RAS on YB-1 phosphorylation induced by ionizing radiation. Breast Cancer Res 13, R28, https://doi.org/10.1186/bcr2845 (2011).

21. Sheridan, C. M. et al. YB-1 and MTA1 protein levels and not DNA or mRNA alterations predict for prostate cancer recurrence. Oncotarget 6, 7470-7480, https://doi.org/10.18632/oncotarget.3477 (2015).

22. Kosnopfel, C., Sinnberg, T. \& Schittek, B. YB1, a prognostic marker and target in tumour therapy. European Journal of Cell Biology 93, 61-70 (2013).

23. McDermott, N. et al. Fractionated radiation exposure amplifies the radioresistant nature of prostate cancer cells. Scientific reports 6 , 34796, https://doi.org/10.1038/srep34796 (2016).

24. Bristow, R. G. \& Hill, R. P. Hypoxia and metabolism. Hypoxia, DNA repair and genetic instability. Nat Rev Cancer 8, 180-192 (2008).

25. Marignol, L., Coffey, M., Lawler, M. \& Hollywood, D. Hypoxia in prostate cancer: a powerful shield against tumour destruction? Cancer Treat Rev 34, 313-327, https://doi.org/10.1016/j.ctrv.2008.01.006 (2008).

26. Brown, J. M. Vasculogenesis: a crucial player in the resistance of solid tumours to radiotherapy. The British journal of radiology 87, 20130686, https://doi.org/10.1259/bjr.20130686 (2014).

27. $\mathrm{Pu}, \mathrm{H}$. et al. PARP-1 regulates epithelial-mesenchymal transition (EMT) in prostate tumorigenesis. Carcinogenesis 35, 2592-2601, https://doi.org/10.1093/carcin/bgu183 (2014).

28. Stark, T. W. et al. Predictive value of epithelial-mesenchymal-transition (EMT) signature and PARP-1 in prostate cancer radioresistance. Prostate 77, 1583-1591, https://doi.org/10.1002/pros.23435 (2017).

29. van de Ven, A. L. et al. Nanoformulation of Olaparib Amplifies PARP Inhibition and Sensitizes PTEN/TP53-Deficient Prostate Cancer to Radiation. Mol Cancer Ther 16, 1279-1289, https://doi.org/10.1158/1535-7163.MCT-16-0740 (2017).

30. Asim, M. et al. Synthetic lethality between androgen receptor signalling and the PARP pathway in prostate cancer. Nat Commun $\mathbf{8}$, 374, https://doi.org/10.1038/s41467-017-00393-y (2017).

31. Khorasani, M. et al. Aberrant expression of miR-141 and nuclear receptor small heterodimer partner in clinical samples of prostate cancer. Cancer Biomark, https://doi.org/10.3233/CBM-170696 (2018).

32. Xiao, J. et al. miR-141 modulates androgen receptor transcriptional activity in human prostate cancer cells through targeting the small heterodimer partner protein. Prostate 72, 1514-1522, https://doi.org/10.1002/pros.22501 (2012).

33. Xu, S., Ge, J., Zhang, Z. \& Zhou, W. miR-141 inhibits prostatic cancer cell proliferation and migration, and induces cell apoptosis via targeting of RUNX1. Oncol Rep 39, 1454-1460, https://doi.org/10.3892/or.2018.6209 (2018).

34. Komarova, E. A. et al. p53 inhibitor pifithrin alpha can suppress heat shock and glucocorticoid signaling pathways. J Biol Chem 278, 15465-15468, https://doi.org/10.1074/jbc.C300011200 (2003).

35. Espinoza, I. \& Miele, L. Notch inhibitors for cancer treatment. Pharmacol Ther 139, 95-110, https://doi.org/10.1016/j. pharmthera.2013.02.003 (2013)

36. Li, Y. et al. Multi-targeted therapy of cancer by niclosamide: A new application for an old drug. Cancer letters 349, 8-14, https://doi. org/10.1016/j.canlet.2014.04.003 (2014).

37. Inoue, I., Matsumoto, K., Yu, Y. \& Bay, B. H. Surmounting chemoresistance by targeting the Y-box binding protein-1. Anat Rec (Hoboken) 295, 215-222, https://doi.org/10.1002/ar.22401 (2012).

38. Meunier, A. et al. Hypoxia regulates Notch-3 mRNA and receptor activation in prostate cancer cells. Heliyon 2, e00104, https://doi. org/10.1016/j.heliyon.2016.e00104 (2016).

39. Inder, S. et al. The Notch-3 receptor: A molecular switch to tumorigenesis? Cancer Treat Rev 60, 69-76, https://doi.org/10.1016/j. ctrv.2017.08.011 (2017).

40. Danza, G. et al. Notch signaling modulates hypoxia-induced neuroendocrine differentiation of human prostate cancer cells. Molecular cancer research: MCR 10, 230-238, https://doi.org/10.1158/1541-7786.MCR-11-0296 (2012).

41. Marignol, L., Rivera-Figueroa, K. \& Lynch, T. \& Hollywood, D. Hypoxia, notch signalling, and prostate cancer. Nature reviews. Urology 10, 405-413, https://doi.org/10.1038/nrurol.2013.110 (2013).

42. Xu, R. et al. Molecular and Clinical Effects of Notch Inhibition in Glioma Patients: A Phase 0/I Trial. Clin Cancer Res, https://doi. org/10.1158/1078-0432.CCR-16-0048 (2016).

43. Farah, E. et al. NOTCH signaling is activated in and contributes to resistance in enzalutamide-resistant prostate cancer cells. J Biol Chem, https://doi.org/10.1074/jbc.RA118.006983 (2019).

44. Wang, X. D. et al. Notch signaling is required for normal prostatic epithelial cell proliferation and differentiation. Dev Biol 290, 66-80, https://doi.org/10.1016/j.ydbio.2005.11.009 (2006).

45. Vallejo, D. M., Caparros, E. \& Dominguez, M. Targeting Notch signalling by the conserved miR-8/200 microRNA family in development and cancer cells. EMBO J 30, 756-769, https://doi.org/10.1038/emboj.2010.358 (2011).

46. Wang, Z. et al. Down-regulation of Notch-1 is associated with Akt and FoxM1 in inducing cell growth inhibition and apoptosis in prostate cancer cells. J Cell Biochem 112, 78-88, https://doi.org/10.1002/jcb.22770 (2011).

47. Shahi, P., Seethammagari, M. R., Valdez, J. M., Xin, L. \& Spencer, D. M. Wnt and Notch Pathways have Interrelated Opposing Roles on Prostate Progenitor Cell Proliferation and Differentiation. Stem Cells, https://doi.org/10.1002/stem.606 (2011). 
48. Yong, T., Sun, A., Henry, M. D., Meyers, S. \& Davis, J. N. Down regulation of CSL activity inhibits cell proliferation in prostate and breast cancer cells. J Cell Biochem 112, 2340-2351, https://doi.org/10.1002/jcb.23157 (2011).

49. Song, S. G., Yu, H. Y., Ma, Y. W., Zhang, F. \& Xu, X. Y. Inhibition on Numb/Notch signal pathway enhances radiosensitivity of lung cancer cell line H358. Tumour Biol, https://doi.org/10.1007/s13277-016-5134-7 (2016).

50. Yahyanejad, S. et al. NOTCH blockade combined with radiation therapy and temozolomide prolongs survival of orthotopic glioblastoma. Oncotarget, https://doi.org/10.18632/oncotarget.9275 (2016).

51. Gimenez-Bonafe, P. et al. YB-1 is upregulated during prostate cancer tumour progression and inreases P-glycoprotein activity. The Prostate 59, 337-349 (2004)

52. Kimira, M., Arai, Y., Shimoi, K. \& Watanabe, S. Japanese intake of flavonoids and isoflavonoids from foods. J Epidemiol 8, 168-175 (1998).

53. Franken, N. A., Rodermond, H. M., Stap, J., Haveman, J. \& van Bree, C. Clonogenic assay of cells in vitro. Nature protocols 1, 2315-2319, https://doi.org/10.1038/nprot.2006.339 (2006).

54. Treindl, F. et al. A bead-based western for high-throughput cellular signal transduction analyses. Nat Commun 7, 12852, https://doi. org/10.1038/ncomms12852 (2016).

55. Schmittgen, T. D. \& Livak, K. J. Analyzing real-time PCR data by the comparative C(T) method. Nature protocols 3, 1101-1108 (2008).

56. Lai, Y. et al. PARP1-siRNA suppresses human prostate cancer cell growth and progression. Oncol Rep 39, 1901-1909, https://doi. org/10.3892/or.2018.6238 (2018).

57. Takayama, K. I., Suzuki, T., Fujimura, T., Takahashi, S. \& Inoue, S. Association of USP10 with G3BP2 Inhibits p53 Signaling and Contributes to Poor Outcome in Prostate Cancer. Molecular cancer research: MCR 16, 846-856, https://doi.org/10.1158/1541-7786. MCR-17-0471 (2018)

58. Idowu, J. et al. Aberrant Regulation of Notch3 Signaling Pathway in Polycystic Kidney Disease. Scientific reports 8, 3340, https://doi. org/10.1038/s41598-018-21132-3 (2018).

59. Wang, T. et al. Fibulin- 4 is associated with prognosis of endometrial cancer patients and inhibits cancer cell invasion and metastasis via Wnt/beta-catenin signaling pathway. Oncotarget 8, 18991-19012, https://doi.org/10.18632/oncotarget.15086 (2017).

60. Heumann, A. et al. Up regulation and nuclear translocation of Y-box binding protein 1 (YB-1) is linked to poor prognosis in ERGnegative prostate cancer. Scientific reports 7, 2056, https://doi.org/10.1038/s41598-017-02279-x (2017).

61. Wang, Y. et al. P21-Activated Kinase Inhibitors FRAX486 and IPA3: Inhibition of Prostate Stromal Cell Growth and Effects on Smooth Muscle Contraction in the Human Prostate. PLoS One 11, e0153312, https://doi.org/10.1371/journal.pone.0153312 (2016).

62. Middleton, R. C. et al. Newt cells secrete extracellular vesicles with therapeutic bioactivity in mammalian cardiomyocytes. J Extracell Vesicles 7, 1456888, https://doi.org/10.1080/20013078.2018.1456888 (2018).

63. Tavares, S. et al. Actin stress fiber organization promotes cell stiffening and proliferation of pre-invasive breast cancer cells. Nat Commun 8, 15237, https://doi.org/10.1038/ncomms15237 (2017).

64. Chin, Y. T. et al. Thyroxine inhibits resveratrol-caused apoptosis by PD-L1 in ovarian cancer cells. Endocr Relat Cancer 25, 533-545, https://doi.org/10.1530/ERC-17-0376 (2018).

\section{Acknowledgements}

We would like to acknowledge support from the Irish Cancer Society (grant code PCA12MAR) and Science Foundation Ireland (grant code 16/TIDA/3991). We also acknowledge the partial financial support by the European H2020 Research Project NOCANTHER (\#685795), AMBER centre (\# SFI/12/RC/2278.) Taylor Jamerson and Angela Nilda Flores were Mount Sinai International Exchange Program minority student participants. Their work was supported in part by grant MD001452 from the National Center on Minority Health and Health Disparities of the National Institutes of Health. Niamh Ni Labhrai was supported by a grant from the M.Sc. in Molecular Medicine, Department of Clinical Medicine at Trinity College Dublin.

\section{Author contributions}

I.S. and N.Mc.D. generated the samples for protein profiling. J.S. and G.E. generated the profiles. I.S., M.B., N.N.L., N.Mc.D., T.J., A.N.F. and A.P.M. contributed to the independent validation of the results. M.B. carried out the immunohistochemistry. D.C. and S.F. reviewed all histopathology slides. P.T. assisted with patient consent. All authors participated in the analysis of the results. L.M. conceived the study and drafted the manuscript. All authors revised the final manuscript.

\section{Competing interests}

J.S. and G.E. are employees of NMI TT Pharmaservices, which provides commercial DigiWest services. The remaining authors declare no potential conflict of interest.

\section{Additional information}

Supplementary information is available for this paper at https://doi.org/10.1038/s41598-019-53799-7.

Correspondence and requests for materials should be addressed to L.M.

Reprints and permissions information is available at www.nature.com/reprints.

Publisher's note Springer Nature remains neutral with regard to jurisdictional claims in published maps and institutional affiliations.

Open Access This article is licensed under a Creative Commons Attribution 4.0 International License, which permits use, sharing, adaptation, distribution and reproduction in any medium or format, as long as you give appropriate credit to the original author(s) and the source, provide a link to the Creative Commons license, and indicate if changes were made. The images or other third party material in this article are included in the article's Creative Commons license, unless indicated otherwise in a credit line to the material. If material is not included in the article's Creative Commons license and your intended use is not permitted by statutory regulation or exceeds the permitted use, you will need to obtain permission directly from the copyright holder. To view a copy of this license, visit http://creativecommons.org/licenses/by/4.0/.

(C) The Author(s) 2019 